\title{
Application of Modified Thermodynamic Grinding Theory in Jet Mill
}

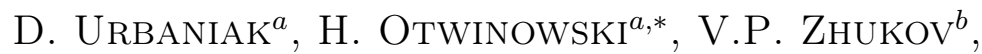 \\ A.Y. BAROCHKIN ${ }^{b}$, J. BORYCA ${ }^{a}$ AND T. WYLECIAE ${ }^{a}$ \\ ${ }^{a}$ Częstochowa University of Technology, 42-201 Częstochowa, Poland \\ ${ }^{b}$ Ivanovo State Power Engineering University, 153003 Ivanovo, Russia
}

Doi: 10.12693/APhysPolA.139.529

*e-mail: otwinowski@imc.pcz.pl

\begin{abstract}
Two problems are particularly important in the works devoted to the grinding process. One of them (the grinding theory) is the dependence that describes the relationship between the energy supplied to the process and its effect. The second issue is the description of particles size distribution of the grinding product. Grinding theories are generally not universal. They relatively reliably describe the effect of cause-and-effect in the crushing process, others - in the grinding process in mechanical mills, and still others - in the superfine comminution process. The so-called thermodynamic theory of grinding is discussed in the present paper. This theory is a combination of Rittinger's theory and Kick's theory. In relation to the classic form of the theory, a certain modification was proposed, resulting from the authors' own research. The results allowing the verification of the proposed modification are presented. In order to analyze the modified form of the thermodynamic theory of grinding, the quantities determining the input of kinetic energy were calculated on the basis of research on the grinding process in a jet mill. The modified thermodynamic grinding theory allows the influence of material defects on the actual value of the resistance to destruction of the ground material to be taken into account. Determining the total energy necessary for grinding by two components - volumetric and surface - allows the influence of both the internal structure of the substance and the external surface of the feed to be taken into account in the grinding process. The validity of this description is confirmed by experimental studies.
\end{abstract}

topics: comminution, grinding energy, thermodynamic grinding theory, jet mill

\section{Introduction}

In the history of research on the grinding process, determining the relationship linking the energy input necessary to carry out the process with the effect of grinding the material appeared as the main area of theoretical and experimental considerations. This relationship is often equated with the notion of the theory of grinding. It has always aroused a great deal of controversy and, as a general rule, it worked only in a narrow range of grinding. Already the first two theories of grinding were opposed. Rittinger [1-3] observed that the grinding energy increases in proportion to the increase in the substance surface. Kick [1-3] argued, however, that this energy is proportional to the volume of the substance, and thus, to its amount.

\section{Theories of grinding}

The number of existing theories regarding the grinding theories is significant. However, two of them deserve special attention and treatment: Rittinger's theory and Kick's theory. They were formulated relatively long ago, while their unwavering significance stems from the fact that most of the theories developed later are either an appropriate combination of both theories, or the development of one of them.

Rittinger's theory (1867) involves energy input with an increase in the external surface of the product. Thus, the grinding work $[\mathrm{J}]$ reads as

$$
L_{n}=l_{0} \Delta A \text {, }
$$

where $l_{0}$ - the work needed to create a unit of surface increment $\left[\mathrm{J} / \mathrm{m}^{2}\right]$ and $\Delta A$ - the increase in the surface of the material in the grinding process $\left[\mathrm{m}^{2}\right]$.

The amount of material and its initial state is omitted in this theory. In addition, the energy of the elastic loads is not taken into account, which means that the validity of this theory is not confirmed by experiments in terms of coarse-grained crushing. If Rittinger's theory was correct in the whole range of grinding, the surface increase of the substance would be the only determinant of grinding energy input. The same amount of energy would produce the same grinding effect both in the case of a fine-grained material and in the case of a coarsegrained material. 
Different trends can be seen in Kick's theory (1885). The energy input is associated only with the total volume of the substance. Thus, the total work of crushing $[\mathrm{J}]$ reads as

$$
L=\frac{\sigma_{m}^{2}}{2 E} V
$$

where $\sigma_{m}$ - the compressive stress on the verge of destruction $\left[\mathrm{N} / \mathrm{m}^{2}\right], E-$ Young's modulus $\left[\mathrm{N} / \mathrm{m}^{2}\right]$, and $V$ - the volume of material $\left[\mathrm{m}^{3}\right]$.

In this theory, the impact of changes in the surface of ground substance on the amount of energy input as well as the state of matter before grinding is omitted. According to this theory, the same energy is needed to grind a very fine material and a very coarse material. Therefore, the same total volume of the substance is the only condition that should be met.

In terms of coarse crushing in low speed machines, studies confirmed the validity of Kick's theory, while Rittinger's theory has been confirmed for fine grinding in the case of high speed machines [4].

\section{Thermodynamic theory of grinding}

The thermodynamic theory of grinding is a combination of a modified Rittinger's theory and the classical Kick's theory [5]. Thus, it can be concluded that this is a model of the phenomenon that applies to the whole range of grinding. The effect of grinding is not identified therein, as in Rittinger's theory, with an increment in the surface of the substance, but with the final surface of the product. Furthermore, the energy supplied in the grinding process in a jet mill is proportional to the total volume of the substance. The initial kinetic energy [J] reads as

$$
E_{k s 1}=\frac{\sigma_{m}^{2}}{2 E} V+\alpha\left(A_{1}+\Delta A\right),
$$

where $\alpha$ the surface density of grinding en$\operatorname{ergy}\left[\mathrm{J} / \mathrm{m}^{2}\right]$, and $A_{1}$ - the surface of the feed $\left[\mathrm{m}^{2}\right]$.

\section{Modified form of thermodynamic theory of grinding}

Resistance to destruction is the stress required to break atomic bonds. In the case of a solid material, the theoretical value of this physical magnitude is determined by its crystal structures. Regular crystals are difficult to observe in the internal structure of brittle materials. Microscopic observations show rather the presence of randomly distributed clusters of crystals, aggregates with an incorrectly distorted spatial form, called crystallites $[6,7]$. In addition, defects in the internal structure of the crystal lattice, along with microcracks, micropores, the inclusions of other minerals or other defects often constitute the natural structure of these materials. The theoretical value of the stress causing the destruction of solid grains is $10^{2} \div 10^{4}$ times greater than the actual strength, as demonstrated by experiments [6].
Griffith [8] postulated that in any solid, there are small cracks (fissures) that weaken its structure. When the appropriate stresses are applied, the fissure increases and the material cracks. According to the concept of the weakest link, the material is torn apart if the local breaking stresses exceed the critical stress.

The remarks above were the reason to propose a modified form of the thermodynamic theory of grinding

$$
E_{k s 1}=\left(\frac{\sigma_{m}^{2}}{2 E}-C\right) V+\alpha A_{2},
$$

where $C$ - the volumetric density of the energy of material weakening as a result of the existence of defects in the internal construction of the substance $\left[\mathrm{J} / \mathrm{m}^{3}\right]$.

The magnitude of $C$ determines the impact of the existing defects in the internal structure of the solid material on the elastic limit strain.

\section{Determination of material constants in grinding process in jet mill}

The nature of the grinding process in this case means that absolute physical magnitudes are not used to describe the process. Rather their streams are responsible for it, namely

$$
\dot{E}_{k s 1}=\left(\frac{\sigma_{m}^{2}}{2 E}-C\right) \dot{V}+\alpha \dot{A}_{2},
$$

where $\dot{E}_{k s 1}$ - the stream of initial kinetic energy of grinding material $[\mathrm{J} / \mathrm{s}], \dot{V}$ - the stream of the volume of material $\left[\mathrm{m}^{3} / \mathrm{s}\right]$, and $\dot{A}_{2}$ - the stream of the total final surface of the ground material $\left[\mathrm{m}^{2} / \mathrm{s}\right]$.

It is assumed that:

$$
\frac{\sigma_{m}^{2}}{2 E}-C=\beta \text {. }
$$

Then,

$$
\dot{E}_{k s 1}=\beta \dot{V}+\alpha \dot{A}_{2} .
$$

To determine the energy input necessary to grind a specific material, the two material characteristics of brittle substances should be designated:

- the surface density of the grinding energy $\alpha$,

- the volumetric density of the grinding energy $\beta$.

The method of determining the material characteristics is based on the condition of maintaining a constant value of the elastic limit load energy streams in individual tests [9]:

$$
\left(\frac{\sigma_{m}^{2}}{2 E}-C\right) \dot{V}=\text { idem. }
$$

The case described by (5) is a straight line in the coordinate system of $\left(\dot{E}_{k s 1}-\dot{A}_{2}\right)$ (Fig. 1) [9].

$$
\begin{aligned}
& \text { If } \dot{A}_{2}=0 \text {, then } \\
& \dot{E}_{k s 1}=\beta \dot{V} \text {. }
\end{aligned}
$$




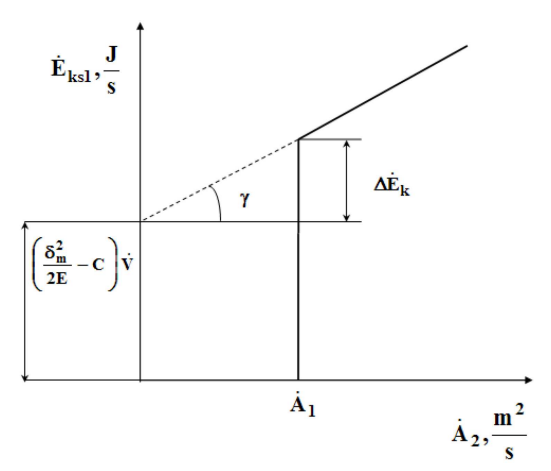

Fig. 1. Scheme to determine material characteristics $\alpha$ and $\beta$.

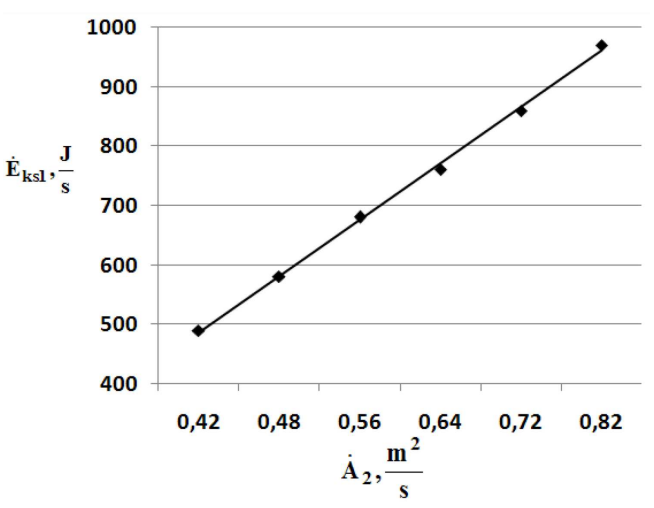

Fig. 2. Relationship between kinetic energy and surface of the grinding product.

Thus, the material size related to the type of substance $\left(\sigma_{m}, E, C\right)$ will be the value of the kinetic energy stream at which the straight line intersects the axis $\dot{E}_{k s 1}$.

Based on Fig. 1 it follows that

$$
\begin{aligned}
& \Delta \dot{E}_{k s}=\dot{E}_{k s 1}\left(\dot{A}_{1}\right)-\dot{E}_{k s 1}(0)= \\
& \alpha \dot{A}_{1}+\left(\frac{\sigma_{m}^{2}}{2 E}-C\right) \dot{V}-\left(\frac{\sigma_{m}^{2}}{2 E}-C\right) \dot{V}=\alpha \dot{A}_{1}
\end{aligned}
$$

and

$$
\tan (\gamma)=\frac{\Delta \dot{E}_{k s 1}}{\dot{A}_{1}}=\frac{\alpha \dot{A}_{1}}{\dot{A}_{1}}=\alpha .
$$

The material magnitude, related to the state and physicochemical properties of the ground material, will therefore be equal to the tangent of the angle of inclination of the straight line $\dot{E}_{k s 1}=f\left(\dot{A}_{2}\right)$.

\section{Implementation of research}

Silica sand with the grain size ranging around $250 \div 630 \mu \mathrm{m}$ was used in the study to analyze the modified form of the thermodynamic theory of grinding. The material was subjected to one-off grinding in the jet mill stand. The tests of grinding were carried out at different values of working air pressure, which determined different values of grinding energy. During the tests, the following parameters were measured: working air pressure and temperature; ambient air humidity, pressure and temperature; as well as time of the measurement. The values of these parameters were used as the input magnitudes of the basic calculation algorithm.

The conducted experimental studies of one-off grinding of sand samples in a jet mill, with a constant volume streams of the ground material, showed that the relationship between the surface stream of the grinding product and the energy necessary for this process is a straight line (Fig. 2). This proves the correctness of the adopted theoretical assumptions.

\section{Conclusions}

The modified form of the thermodynamic theory of grinding allows the impact of defects in materials on the actual value of resistance to destruction of the ground material to be emphasized. Determining the total energy required for grinding by two components - volumetric and surface - allows the impact of both the internal structure of the substance and the external surface of the feed to be taken into account in the grinding process. The validity of such a description is confirmed by experimental studies.

The conducted research confirmed the assumption that while maintaining the constant stream of the ground material during the tests, the dependence of the energy stream as a function of the product surface stream in the form of a straight line is obtained.

\section{References}

[1] G.M. Barrow, Physical Chemistry, PWN, Warszawa 1973 (in Polish).

[2] J.G. Bennet, J. Inst. Fuel 10, 22 (1936).

[3] F.C. Bond, Trans. AIME Mining Eng. 193, 484 (1952).

[4] J.-P. Duroudier, Size Reduction of Divided Solids, ISTE Press Elsevier, London 2016.

[5] E. Mielczarek, Free Grinding of Brittle Solids, Częstochowa University of Technology Scientific Papers, No. 25, Częstochowa 1982 (in Polish).

[6] J. Zawada, Introduction to the Mechanics of Crushing Processes, Publishing and Printing House of the Institute for Sustainable Technologies, Radom 1998 (in Polish).

[7] S. Jiang, C. Tang, X. Li, Y. Tan, R. Peng, D. Yang, S. Liu, Int. J. Adv. Manuf. Technol. 109, 2795 (2020).

[8] A.A. Griffith, Phil. Trans. Roy. Soc. A 221, 163 (1928).

[9] D. Urbaniak, Scientific Works of the Warsaw University of Technology. Conferences 4, 1283 (2002). 